\title{
Clinical management of smoking cessation: patient factors affecting a reward-based approach
}

\author{
This article was published in the following Dove Press journal: \\ Patient Preference and Adherence \\ 9 December 2010 \\ Number of times this article has been viewed
}

\author{
Jeanette M Renaud' \\ Michael T Halpern² \\ 'RTI International, Research Triangle \\ Park, NC, USA; ${ }^{2}$ RTI International, \\ Washington, DC, USA
}

Correspondence: Jeanette Renaud RTI International, 3040 East

Cornwallis Road, Research

Triangle Park NC 27709, USA

$\mathrm{Tel}+\mathrm{I}$ 919-316-3770

Fax + | 9|9-54|-6683

Email jrenaud@rti.org
Abstract: Although the majority of current smokers indicate they would like to quit, only about half of smokers make a quit attempt each year. Of those who attempt to quit, only about $5 \%$ are successful. Many effective products and programs are available to assist in smoking cessation; however those interested in quitting often do not make use of these resources. To increase use of cessation products in order to improve successful cessation rates, the Consumer Demand Roundtable has argued that smokers need to be viewed as consumers of cessation products rather than as patients needing treatment. With this consumer-based approach in mind, the current review examines how participant characteristics, perceptions, and behavior influence, and are influenced by, contingency management (CM) paradigms in various settings. Findings suggest that participant factors associated with success in these programs include demographic characteristics (eg, gender, marital status), self-efficacy, motivation to quit, and impulsivity. Overall, participants perceive incentives for successful cessation as motivating. However, such programs may involve greater withdrawal symptoms (eg, craving for cigarettes) initially, but these symptoms tend to decrease at a greater rate over time compared with nonincentive group participants. CM programs have also been shown to be successful across a number of settings (eg, communities, schools), including settings in which smokers are often considered difficult to treat (eg, substance abuse treatment centers). Overall, CM programs are perceived positively by participants and can increase rates of successful cessation. Furthermore, CM interventions have the flexibility to adapt to individual preferences and needs, leading to greater participation and likelihood of successful cessation. Thus, CM provides an important framework for addressing the need for consumer-focused smoking cessation interventions.

Keywords: smoking cessation, tobacco cessation, contingency management, reward, incentive, perception

\section{Introduction}

Smoking is both physiologically and psychologically addictive, making it extremely difficult to quit even if the desire to do so is strong. Approximately $70 \%$ of those who smoke indicate that they would like to quit. ${ }^{1}$ However, among the more than $40 \%$ of smokers who do make a quit attempt each year, ${ }^{2}$ only about $5 \%$ experience long-term (3-12 months) success. ${ }^{1,3}$ The methods used for attempting to quit smoking include selfhelp, counseling, and pharmacologic interventions. Self-help methods include unaided quitting and use of smoking substitutes, such as gum or candy. Counseling for smoking cessation ranges from intensive, in-person individual counseling, to group sessions, to counseling provided via other means (eg, quitlines). Pharmacologic interventions include nicotine replacement therapy (NRT) as well as non-nicotine medications that assist with symptoms experienced during cessation (eg, bupropion). Combinations of 
these methods (eg, counseling and NRT) are more successful than any strategy alone, and multiple quit attempts are often required in order to achieve success. ${ }^{4}$

However, although many products and programs to assist smokers in quitting are available, relatively few of those interested in quitting consider or utilize these options. Only about one-third of quit attempts involve the use of any of the treatment options available. ${ }^{5}$ Thus, part of the lack of success may be due to underutilization of the options available to aid in cessation. Recently, the National Tobacco Cessation Collaborative (NTCC) and its Consumer Demand Roundtable (CDR) advocated a consumer-based approach to smoking cessation treatment, whereby smokers are viewed as "consumers" instead of as "patients," is needed in order to increase awareness, demand, and use of cessation products and programs.

One consumer-focused approach to cessation is based on the psychological principle of operant conditioning, which provides rewards in response to desired behaviors. As part of smoking cessation, these strategies, also known as contingency management (CM), involve offering rewards such as money or vouchers, to program participants for successful cessation. In these programs, successful abstinence may be determined via self-report or by biochemical tests (eg, carbon monoxide [CO] levels), and may be assessed once (eg, end of an intervention) or at multiple time points over time (eg, throughout an intervention).

A recent review article ${ }^{6}$ summarized the efficacy of $\mathrm{CM}$ strategies in smoking cessation. The current review takes a consumer-based approach to understand how participant characteristics, perceptions, and behaviors influence and are influenced by CM paradigms in various settings.

\section{Methods}

A Medline search was conducted during April 2010 (a follow-up search was conducted in August 2010) to identify articles examining the use of contingency management for smoking cessation interventions. The primary search was done using the following parameters: "smoking cessation [MESH] AND ('contingency management' OR 'reward' OR 'incentive')". Searches were limited to articles published in English, focused on humans, and published between 2000 and 2010. The search resulted in 166 articles. The abstract for each of the retrieved articles was reviewed to determine if a full review of the article was warranted. As a result of this process, 54 articles were excluded from further review. Excluded articles included: 16 focused on provider (rather than patient) pay-for-performance; 19 involved biological or clinical assessments (eg, pharmacology, psychopharmacology, neurology, neurobiology, genetics) related to nicotine addiction; 11 focused on methodology (eg, recruitment, surveys, models); 5 included unique population samples (eg, renal transplant and foot surgery patients) which make their results less generalizable; and 3 examined nicotine as the reward itself.

Full-text articles for the remaining 112 citations were retrieved for closer review to determine if the article involved a CM paradigm and included any information related to patient characteristics and perceptions. Two individuals reviewed each of the articles, and excluded an additional 80 articles. About half of these articles were excluded for similar reasons to those used for exclusion following review of the abstracts. For instance, 31 involved an examination of biological or clinical aspects of smoking, 7 examined nicotine as a reward for smokers, 2 involved tobacco addiction in relation to weight-related concerns, 1 focused on methodological issues, and 1 involved a specialized (less generalizable) population sample. Thirty-eight articles did involve a CM program intervention, but did not include any measures or discussion of patient characteristics or perceptions.

The remaining 32 articles included information relevant to the current review. An additional 11 articles were identified from the reference sections of 2 review articles included in the initial search, and 4 of these were subsequently excluded. Thus, 39 articles included information relevant to patient factors influencing the success of reward-based approaches to smoking cessation and are therefore included in the findings.

\section{Findings Types of programs}

Incentives in smoking cessation programs have been used in several different paradigms. For instance, quit and win programs $^{7-11}$ offer the chance to win large monetary or voucher prizes among smokers who enter cessation programs or are successful with such programs. These programs are similar to lotteries in which a large number of people may enter but only a few actually receive prizes. In contrast, treatment cost reimbursement programs ${ }^{12-14}$ provide reimbursements for the costs of cessation program for all participating smokers if they are successful. These reimbursement programs require smokers initially to pay for their treatment programs, and provide reimbursement only if they successfully complete the programs. In other types of CM programs, instead of the possibility of winning a large prize (as in a quit to win program), participants are guaranteed smaller rewards 
(eg, money, vouchers $)^{9,15-17}$ at multiple time points based on objective evidence of abstinence during a (typically brief) specified time period. If a participant is not successful at a particular time point, he/she will not receive payment at that time, but may continue in the program and earn rewards at subsequent time points if successful in abstaining. Thus, these programs offer more potential rewards to smokers if they relapse briefly during the program.

\section{Participant characteristics \\ Characteristics of participants in CM cessation programs}

Demographic characteristics of those who participated in community-based quit and win programs were assessed in 3 studies. Among participants in an annual quit and win program sponsored by a county Health Department in Kentucky, most participants were white, educated at least at the high school level, had been smokers for more than a decade, and had attempted to quit on multiple occasions. ${ }^{9}$ Another study compared characteristics of those who entered quit and win contests in 11 communities across New York to characteristics of smokers in the same regions of the state. ${ }^{10}$ They found that those who entered the quit and win contests tended to be younger and smoke more cigarettes per day.

Hawk and colleagues ${ }^{11}$ evaluated participant characteristics in two programs, one that used the possibility of winning a large prize to motivate smokers to quit (ie, quit and win) and the other provided smokers with a nicotine replacement therapy (NRT) voucher to mitigate a potential barrier to quitting (ie, cost). Those who participated in the quit and win program without requesting the NRT voucher were younger on average than those who requested the NRT voucher alone. Further, in comparison with characteristics of the overall smoking population in the region, those who participated in either program were more educated and heavier smokers. Both programs were equally effective in recruiting participants, and quit rates were similar across both programs. Those who participated in both programs were no more successful than those who participated in only one of the programs.

\section{Participant characteristics associated with success or failure in CM cessation programs}

A number of demographic characteristics have also been shown to be related to cessation success in CM programs, including gender (male), age (older), socioeconomic status (higher), race (white), and marital status (married). A study by Amodei and Lamb ${ }^{18}$ examined demographic characteristics related to success in a CM program based on whether participants indicated that they used NRT to aid in quitting. These researchers found use of NRT on the first day of the study was the most reliable predictor of initial abstinence (ie, first 10 days of cessation attempt). Both self-efficacy and NRT use were significant predictors of the total number of days abstinent in a multivariate model. They also found that those who were married or in a domestic partnership were more than 3 times as likely to have at least 1 day of abstinence during the first 10 days of the study. Associations between gender or race and successful cessation were significant when examined in univariate analyses. However, these relationships did not remain significant when examined in multivariate regression models that included marital status, self-efficacy, intentions to quit, NRT use, and stages of change.

Other studies have also explored the relationship between self-efficacy and motivation to quit with successful cessation in CM programs. For instance, Romanowich et $\mathrm{al}^{19}$ found that providing rewards for abstinence led to greater self-efficacy, rather than greater self-efficacy leading to successful abstinence in a CM program. Thus, the provision of rewards may increase self-efficacy, which in turn increases successful abstinence. Heil and colleagues ${ }^{20}$ assessed whether monetary rewards could motivate smokers not currently trying to quit smoking. These researchers included three groups of participants in a cessation program for 5 days. For 2 of the groups, payment was contingent upon abstinence, while the third group was paid regardless of abstinence. Interestingly, they found that monetary rewards were effective in motivating those not previously intending to quit smoking when rewards were contingent upon proof of abstinence, regardless of the amount of the reward. Thus, rewards may be effective in motivating smoking cessation in and of itself, regardless of one's intention or desire to quit.

\section{Time preference for smoking cessation rewards}

The extent to which individuals discount future rewards in favor of smaller immediate rewards (ie, has a time preference for short-term rewards) has been used as a behavioral economic index of impulsivity. This time preference for short-term rewards is associated with a variety of smoking behaviors, such as the likelihood of smoking among adults and increased nicotine use (eg, number of cigarettes smoked per day) among smokers. ${ }^{21}$ Ohmura and colleagues ${ }^{22}$ suggest that while heavier smokers (ie, 20 or more cigarettes per day) may be more likely to discount delayed rewards than do never smokers, lighter smokers (less than 20 cigarettes 
per day) did not differ in their time preference compared with never smokers.

The finding that heavier smokers are more likely to discount delayed rewards suggests that the schedule of reinforcement (ie, the time from change in behavior to receipt of reward) used in CM programs may have a great impact on successful cessation. ${ }^{23}$ Heavier smokers may be more successful at abstinence if shorter reward schedules are in place. The direction of reinforcement may also be important for successful cessation among heavier smokers. Mueller et a ${ }^{24}$ rewarded abstinence for some participants by increasing the amount received as the length of abstinence increased. Participants in this "increasing group" received about US\$8 in the first minute of successful abstinence and earned about 21 cents for each subsequent minute-long segment in which they remained abstinent for a potential total of about US\$32. Other participants were provided the same maximum reward upfront, but the amount was reduced as the length of abstinence was reduced. This "decreasing group" received about US\$32 for the first minute of abstinence, but the amount decreased by 21 cents for each unsuccessful period of abstinence resulting in a potential total of about US\$8 if they were consistently unsuccessful. The remaining participants were rewarded the same amount of money at each successful interval. Those in the increasing reward condition were most successful in abstaining from smoking during the 2-hour session. Furthermore, similar to the results of Ohmura et al, ${ }^{22}$ the number of cigarettes smoked per day was a strong predictor of successful abstinence, with heavier smokers being less likely to maintain abstinence.

MacKillop and Kahler ${ }^{25}$ sought to clarify whether greater discounting of delayed rewards is a predictor of successful smoking cessation (ie, does an individual's preference for delayed rewards prior to a quit attempt predict cessation) or a product of attempting cessation (ie, does an individual's preference for delayed rewards change during quit attempts). To address this issue, they measured time preference for rewards (ie, the degree to which delayed rewards were discounted) prospectively in a group of current smokers wanting to quit. They found that those who exhibited more short-term time preference in a delayed discounting measure prior to cessation attempts were more likely to relapse than were their counterparts with longer-time preferences. This finding remained significant in analyses where the effect of nicotine dependence was held constant suggesting that impulsivity in and of itself plays an important role in successful cessation.
Among a group of adolescent smokers, Krishnan-Sarin et $\mathrm{a}^{26}$ assessed impulsivity using both self-report and behavioral measures prior to cessation attempts. They found that behavioral measures of impulsivity (ie, greater discounting of delayed rewards) were more predictive of lack of continuous success in smoking cessation than were self-report measures. That is, adolescents who behaved impulsively were more likely to relapse than adolescents who behaved less impulsively, but there was less association between self-reported level of impulsivity and success in smoking cessation. Krishnan-Sarin et a ${ }^{26}$ suggest that the differences in self-report and behavioral measures of impulsivity reflect differences between more general trait-like impulsivity (ie, an individual's level of impulsivity or time preference being relatively consistent across time and situations) and specific behavioral responses to a situation (ie, an individual's level of impulsivity/time preference being influenced by the current situation).

In addition to nicotine dependence, both income and educational attainment have been found to be associated with time preferences for rewards. MacKillop and $\mathrm{Kahler}^{25}$ found a positive relationship between income and preference for larger, delayed rewards. Jaroni et $\mathrm{al}^{27}$ found that those who were less educated were more likely to discount delayed rewards than were those who were more educated.

Because time preference for short-term rewards decreases the likelihood of successful cessation, a different reward schedule may be useful for these individuals. For example, a study by Heil et $\mathrm{al}^{28}$ suggests that rewarding participants based on abstinence at later points in a cessation program does not improve success over requiring continued abstinence over the entire program. In this study, for half of the participants (contingent-all), payment was contingent upon evidence of abstinence in all 3 time periods, while the other half of participants (contingent-last) received rewards contingent upon abstinence in the last period, regardless of abstinence in the first two periods. Those in the contingentall condition experienced higher levels of abstinence in all 3 periods, including the final period. This suggests that a CM framework requiring strict abstinence may be more successful than a program which allows behaviors towards cessation (ie, incomplete abstinence) and assessment of abstinence only at the end.

\section{Participant perceptions}

Perceptions of rewards

Although focused on CM for drug addiction, a study by Roll and Chudzynski ${ }^{29}$ shows the importance of understanding 
participant perceptions of rewards offered in CM programs. As part of this study, researchers asked both staff and patients in a drug treatment center to rate a series of rewards in terms of how reinforcing they believed them to be. Overall, the ratings between staff and patients were comparable. However, staff perceived graduation ceremonies, tokens, and free meals significantly more reinforcing than did patients, and General Education Development classes at the clinic, legal advice, and financial advice as less reinforcing than did patients. Thus, it seems patients prefer rewards that may be more meaningful and lasting (eg, meeting with a financial advisor) than those that are more celebratory but fleeting (eg, pizza parties). Further, perceptions of the impacts of CM rewards may differ between intervention designers/administrators and participants.

Two studies asked participants whether the rewards they received had motivated them to refrain from smoking during an intervention. In one study, 53 adolescents who attended the final treatment session ( 10 weeks after the intervention began) filled out an "Impressions of Treatment Questionnaire". ${ }^{30}$ When asked what aspects of the program they found to be most helpful, a majority (88.7\%) indicated that earning points for abstinence (assessed via a biological measure) at each session that could be exchanged for gift certificates was helpful. Another study ${ }^{31}$ involved a cessation program that rewarded those who remained abstinent for 1 year or longer following their intervention with a diploma and a "former smoker" pin at a short ceremony. To assess participants' perceptions of the rewards, 100 former smokers who received the diploma and pin were asked to respond to a brief questionnaire. All respondents indicated that they valued the rewards, with more respondents preferring the diploma to the pin. Respondents indicated that the diploma represented an official acknowledgment of their success. The pin was a way to get attention from others and feel pride in their selves. Some felt it was also a way to be an example to other smokers. However, most of these respondents reported that the rewards did not add to their ability to maintain abstinence.

Focus groups were used in 2 studies to assess potential smoking cessation program participants' perceptions of different types of interventions, including rewards. One of these studies ${ }^{32}$ included focus groups with smokers at a number of worksites to assess perceptions of how employers could assist with smoking cessation. Choosing from a provided list of possible cessation interventions, a majority of employee respondents felt that incentives and quit smoking contests could help with successful cessation. However, some employees thought incentives for smoking cessation would be unfair to nonsmokers and may be too costly and difficult for their employers to implement.

Another study ${ }^{33}$ involved focus groups with adolescent smokers. Although these participants initially expressed difficulty imagining being motivated by incentives to stop smoking, when pressed most suggested money as a potential reward. Suggested amounts of money ranged from very large sums (thousands of dollars) to more realistic amounts of about US\$10 per week. Other incentives mentioned included cars, trips, and tickets to games or concerts. Participants were also asked what types of incentives might encourage adolescent smokers to join and continue with a cessation program. In a school-based setting, missing a class was perceived as a reward in and of itself. Other suggestions included providing food such as pizza or cake.

\section{CM program effects on participants Effects of CM on self-reported craving and desire to quit smoking}

Individuals' level of craving and desire to quit smoking are affected differently as a function of CM condition. Alessi et $\mathrm{al}^{34}$ had participants who were assigned to $\mathrm{CM}$ or control groups complete self-report measures of nicotine withdrawal symptoms (Minnesota Nicotine Withdrawal Scale, ${ }^{35,36}$ craving (Tiffany Questionnaire on Smoking Urges), ${ }^{37}$ and abstinence self-efficacy and ease of abstinence (both rated along continuums ranging from "not at all" to "extremely"). Those in the CM group reported greater overall ease with abstinence than did the control group. This effect varied as a function of time such that abstinence became easier for those in the CM group while abstinence was reported to be more difficult for those in the control group over the course of the 12-day study. Self-reported abstinence self-efficacy and craving also varied as a function of time, with those in the CM group reporting greater self-efficacy and less craving over time while those in the control group reporting self-efficacy and cravings that were relatively stable over time. Thus, providing monetary incentives to quit smoking not only motivates smokers to quit, but also makes certain aspects of the experience of quitting less severe and, in turn, increases the likelihood of success.

However, not all symptoms associated with cessation appear to decrease among individuals in CM programs. Alessi et $\mathrm{al}^{34}$ found that those in the CM group reported greater withdrawal symptoms than did those in the control group. This effect varied as a function of time; those in the CM group perceived decreasing withdrawal symptoms over time, while withdrawal symptoms remained consistent over 
time for the control group. Other studies ${ }^{17,20}$ have reported that participants (adult and adolescent) receiving incentives for evidence of abstinence reported greater withdrawal, cravings, anger, anxiety, and restlessness than did those receiving incentives independent of abstinence (eg, rewards based only on attendance). Similar to the findings by Alessi et al, ${ }^{34}$ these symptoms did decrease over time. These findings may reflect greater focus on abstaining from smoking among CM group participants motivated by the reward offered, and thus greater focus on the symptoms presenting potential challenges to abstinence.

CM programs may also affect motivations among individuals not yet ready to quit. For instance, Tevyaw et $\mathrm{al}^{38}$ found increases in readiness to quit smoking among those who were provided rewards for successful cessation, half of whom initially indicated no desire to quit, compared with those who were not rewarded for successful cessation. Lamb et $\mathrm{al}^{23}$ used a shaping paradigm whereby participants were rewarded for changes in a target behavior (eg, reductions in cigarette smoking) rather than just for achieving the target behavior (complete cessation) itself. Over the approximately 4 months of the study, participants received rewards for evidence of improvements toward abstinence (ie, decreased CO levels). They found that shaping was an effective method for increasing abstinence. In a related study of individuals who indicated that they have no concrete plans to quit smoking in the next 6 months ("complacent smokers"), Lamb et al ${ }^{39}$ found that providing incentives for evidence of reduced smoking (ie, lower CO levels at each time point compared with the previous time point) led to self-reported greater readiness to quit and greater self-efficacy for abstinence over 3 months. Providing incentives for decreased CO levels among complacent smokers was also associated with fewer cigarettes smoked and more abstinent days over 3 months. ${ }^{40}$ In addition, hard-to-treat smokers (those who were never abstinent during a 10-visit study baseline) were more likely to achieve abstinence in a contingency management with shaping intervention compared with those in an intervention involving contingency management alone. ${ }^{41}$ These findings indicate that providing incentives for reductions in smoking over time may ultimately assist with successful cessation. Smoking cessation programs that rely on all-ornothing strategies may actually impede cessation for some participants.

\section{Effects of CM on program attendance}

Two studies ${ }^{42,43}$ reported that treatment group participants who received rewards for evidence of abstinence also had better attendance in program sessions than the control group participants who were not rewarded.

\section{Specific CM program settings}

Studies of CM interventions have involved a number of different population settings. Settings discussed in this section include communities, schools, worksites, Internet, and mental and substance abuse treatment centers.

\section{Community-based CM programs}

The most common type of CM program implemented at the community level is the quit and win contest. These programs are attractive to communities because they are relatively low cost and can reach large numbers of people through promotion and media. In a review of community-based quit and win contests, Cahill and Perera ${ }^{44}$ concluded that while some of these programs did result in higher long-term quit rates among individuals, the impact at the community level is low (ie, less than 1 in 500 smokers achieves success). They also note that because assessments of abstinence in these programs rely on self-report rather than objective verification of cessation, the actual number of quitters may be even lower than estimated. However, incorporating objective assessments of cessation (eg, biochemical tests) would be difficult and costly, which would make them much less attractive and much less sustainable for many communities. ${ }^{45}$ Thus, the actual effectiveness of quit and win contests is unclear, and it seems unlikely that a clearer assessment of their effectiveness will be obtained any time soon.

\section{School-based CM programs}

With a smaller population size and a common location at which participants spend a large amount of time, schoolbased CM programs allow for more objective assessments of abstinence. As noted previously, being able to miss a class is perceived as a reward in and of itself $f^{33}$ and therefore can result in greater rates of attendance at scheduled abstinence assessment sessions. ${ }^{26}$ Furthermore, school-based CM programs are better able to incorporate educational, counseling, and objective assessment components for which participants are likely to show up, which in turn likely leads to greater program success. ${ }^{6}$

\section{Worksite CM programs}

Worksite CM interventions include some of the same advantages as school-based CM interventions; in particular, the participants spend a large amount of time in a common location. This makes it easier to schedule sessions that may 
be more likely to be attended, and participants may benefit from social support from co-workers. Leeks et al, ${ }^{46}$ in their review of worksite cessation interventions for the Guide to Community Preventive Services, concluded that worksite interventions that combine $\mathrm{CM}$ with other cessation support services, such as counseling and education, are effective in reducing tobacco use among employees. However, they did note that there was insufficient evidence to determine the effectiveness of implementing a CM program alone.

Volpp et $\mathrm{al}^{47}$ worked with a large national corporation to implement a smoking cessation program that offered some employees the opportunity to earn up to US\$750 for quitting smoking. Other employees were given the same cessation information but were not offered the reward. They found that those offered the incentive were 3 times more likely to enroll in the program and be abstinent at the 12- and 18-month follow-up assessments than those not offered an incentive. Other worksite CM cessation studies ${ }^{48,49}$ found increased enrollment, as well as short-term (ie, 2-3 months) cessation rates, among those offered incentives for program attendance and successful cessation. Longer term cessation rates (ie, 6 months or more) in these studies, however, did not differ as a function of incentives.

\section{Internet-based CM programs}

To determine the feasibility and efficacy of employing a CM cessation program via the Internet, Dallery et al $^{16}$ offered participants vouchers that could be used with a small group of Internet merchandise vendors. To obtain objective evidence of abstinence, participants were loaned a laptop, Web camera, and $\mathrm{CO}$ monitor. Twice daily for 4 weeks participants tested their CO levels while being recorded via the Web camera; the video was sent electronically to the researchers. They found that participants were more successful at abstinence when they were able to earn vouchers for their abstinence. Reynolds et $\mathrm{a}^{50}$ found similar results using a similar methodology with adolescent participants. This suggests that not only is it possible to obtain objective assessments of abstinence via the Internet, but also that providing incentives can improve abstinence.

\section{Mental health and substance abuse treatment center CM programs}

Encouraging those currently receiving treatment for substance abuse to quit smoking as well is particularly challenging. However, there is evidence to suggest that $\mathrm{CM}$ can be an effective motivator for this group. Alessi et $\mathrm{al}^{51}$ implemented a CM program in a residential substance abuse treatment center for men. Participants in the CM condition were able to earn small prizes for evidence of abstinence (via cotinine testing). Those with negative results (ie, evidence of abstinence) were given the opportunity to pick a card from a bowl, with corresponding prizes ranging from values of US\$1 (eg, toiletries) to US\$100 (eg, stereo). They found that those in the CM condition had a greater percentage of negative test results overall and a higher number of consecutive days abstinent. A similar study ${ }^{52}$ conducted in a women's residential treatment center found similar results.

Mental health patients provide challenges similar to substance abuse patients. A strong association between smoking and mental health has been reported, ${ }^{53}$ with estimates indicating those with mental illness are nearly twice as likely to smoke as those with no mental illness. ${ }^{54}$ Tidey et $\mathrm{al}^{55}$ conducted a $\mathrm{CM}$ intervention for individuals with schizophrenia at an outpatient mental health center. Similar to the studies discussed above, they found that participants were more successful at abstaining from cigarettes when they received payment for doing so.

\section{Discussion}

Prior research has shown that CM can be an effective strategy for smoking cessation interventions across a variety of settings. ${ }^{6}$ The current review examined CM programs from the perspective of the participants of such programs, in line with a recent statement from the NTCC CDR advocating for a greater consumer-based focus in smoking cessation. ${ }^{5}$ In particular, we have examined characteristics, perceptions, and behaviors of smokers that affect the success of $\mathrm{CM}$ approaches to cessation. Because CM by definition is consumer-focused, it holds tremendous promise for overcoming the current gaps in smoking cessation treatment utilization described by Shiffman. ${ }^{5}$ However, this can be achieved only if $\mathrm{CM}$ interventions apply information from previous studies to develop cessation programs that are more tailored to the individual consumer.

One psychological factor particularly relevant to CMbased interventions is time preference. There is an extensive literature in health economics and related fields examining consumers' time preference for health outcomes and the related concept of discount rates (ie, the amount that health or monetary outcomes occurring in the future are de-valued compared with outcomes occurring in the present). Overall, this literature indicates that individuals with a greater time preference for the present are more likely to engage in unhealthy behaviors and less likely to use preventive care services. For example, Axon et $\mathrm{al}^{56}$ reported that individuals 
with hypertension who have higher discount rates (ie, greater likelihood of discounting future health benefits and thus time preferences for shorter-term outcomes) are less likely to check their blood pressure, alter diet and exercise habits, and see physicians for care when ill. A greater time preference for the present is also associated with increased body mass index, particularly for males ${ }^{57}$ In contrast, women who place a relative greater value on future (versus present) benefits are more likely to use genetic counseling for BRCA1/2, genes indicating increased risk for breast cancer. ${ }^{58}$

Consideration of time preference is important for the development of smoking cessation interventions. Smokers tend to show greater impulsivity and may be more presentoriented than nonsmokers. ${ }^{59}$ Further, preference for shorterterm rewards (as opposed to larger but delayed rewards) is associated with increased likelihood of smoking and with smoking intensity. ${ }^{21,22,60,61}$ Smoking cessation involves accepting short-term costs (in emotional/mental health and quality of life, as well as possible monetary costs) in exchange for long-term benefits. In the studies we reviewed, greater short-term preference (ie, greater discounting of delayed rewards) was associated with decreased likelihood of successful cessation. This has implications for the design of CM cessation interventions. Smokers with stronger shortterm time preferences would more likely benefit from a series of rewards (for cessation) occurring frequently at short time intervals. In contrast, those with longer-term time preferences would likely be optimally motivated by a single larger reward available after a period of continued abstinence. By adjusting the reward frequency to the time preference of an individual smoker, CM approaches to cessation can adopt a greater consumer focus and likely achieve increased rates of successful cessation.

Another consumer-based approach that can be used with $\mathrm{CM}$ cessation interventions is identification of rewards that are most meaningful to the smoker attempting to quit. As indicated by our literature review, smokers' preferences for different types of rewards varied. We are not aware of any $\mathrm{CM}$ cessation interventions that allow smokers to specify or select their (potential) reward at the start of the intervention. However, this approach could result in greater motivation for cessation and thus increased intervention success.

CM interventions may also address desires by (smoking) consumers for a gradual cessation process. As discussed in this review, CM interventions can reduce levels of tobacco consumption in the absence of full cessation as well as increase participation and attendance to program sessions and cessation assessments. Further, participation in CM interventions may lead to increased self-efficacy and motivation for smoking cessation. These aspects of CM interventions have the potential to increase smoker's willingness to participate in future quit attempts and may increase the likelihood of subsequent successful cessation.

One drawback to CM interventions is the greater withdrawal symptoms experienced by those in incentive-based conditions versus those in nonincentive conditions. However, it is important to point out that the experience of greater withdrawal reported by CM participants may be due to their increased motivation and focus on the need (or desire) to abstain from smoking compared with their control group counterparts. Furthermore, while these symptoms are initially greater for CM participants they tend to decrease over time, whereas symptoms among the control group remain relatively stable over time.

Overall, the available literature suggests an opportunity for applying an increased consumer-based focus to $\mathrm{CM}$ cessation interventions. By adapting such interventions to individual preferences, smokers are more likely to participate and more likely to achieve successful cessation. CM is ideal for this purpose and can provide an important framework for addressing the need for consumer-focused smoking cessation interventions. The primary limitation of the current review is that CM studies do not always assess the role of participant factors in successful versus unsuccessful cessation. Furthermore, when participant factors are assessed they are typically secondary or tertiary foci of the research, and therefore are not often explored and discussed fully. As such, the current review relied on information available from a relatively small number of $\mathrm{CM}$ studies in the literature.

\section{Disclosure}

The authors disclose no conflicts of interest in this work.

\section{References}

1. Center for Disease Control and Prevention. Cigarette smoking among adults - United States, 2000. MMWR Morb Mortal Wkly Rep. 2002; 51(29):642-645.

2. Center for Disease Control and Prevention. Tobacco use among adults - United States, 2005. MMWR Morb Mortal Wkly Rep. 2006; 55(42):1145-1148.

3. Hughes JR, Keely J, Naud S. Shape of the relapse curve and longterm abstinence among untreated smokers. Addiction. 2004;99(1): 29-38.

4. Fiore MC, Jaen CR, Baker TB, et al. Treating tobacco use and dependence: 2008 Update. Clinical Practice Guideline. Rockville MD: US Department of Health and Human Services (USDHHS): Public Health Service, 2008.

5. Shiffman S, Brockwell SE, Pillitteri JL, Gitchell JE. Individual differences in adoption of treatment for smoking cessation: Demographic and smoking history characteristics. Drug Alcohol Depend. 2008; 93(1-2):121-131. 
6. Ledgerwood DM. Contingency management for smoking cessation: Where do we go from here? Curr Drug Abuse Rev. 2008;1(3):340-349.

7. Cahill K, Perera R. Quit and Win contests for smoking cessation. Cochrane Database Syst Rev. 2008;(4):CD004986.

8. Hey K, Perera R. Competitions and incentives for smoking cessation. Cochrane Database Syst Rev. 2005:18(2):CD004307.

9. Ryan M, Romanelli F, Nee JK, et al. Characteristics of participants enrolled in a Quit and Win smoking cessation program. J Ky Med Assoc. 2006;104(10):469-474.

10. O'Connor R, Fix B, Celestino P, et al. Financial incentives to promote smoking cessation: Evidence from 11 quit and win contests. J Public Health Manag Pract. 2006;12(1):44-51.

11. Hawk LW Jr, Higbee C, Hyland A, et al. Concurrent quit and win and nicotine replacement therapy voucher giveaway programs: Participant characteristics and predictors of smoking abstinence. J Public Health Manag Pract. 2006;12(1):52-59.

12. Joyce GF, Niaura R, Maglione M, et al. The effectiveness of covering smoking cessation services for Medicare beneficiaries. Health Serv Res. 2008;43(6):2106-2123.

13. Kaper J, Wagena EJ, Willemsen MC, et al. A randomized controlled trial to assess the effects of reimbursing the costs of smoking cessation therapy on sustained abstinence. Addiction. 2006;101(11):1656-1661.

14. Reda AA, Kaper J, Fikrelter H, et al. Healthcare financing systems for increasing the use of tobacco dependence treatment. Cochrane Database Syst Rev. 2009;2:CD004305.

15. Cavallo DA, Cooney JL, Duhig AM, et al. Combining cognitive behavioral therapy with contingency management for smoking cessation in adolescent smokers: A preliminary comparison of two different CBT formats. Am J Addict. 2007;16(6):468-474.

16. Dallery J, Glenn IM, Raiff BR. An Internet-based abstinence reinforcement treatment for cigarette smoking. Drug Alcohol Depend. 2007;86(2-3):230-238.

17. Roll JM. Assessing the feasibility of using contingency management to modify cigarette smoking by adolescents. J Appl Behav Anal. 2005;38(4):463-467.

18. Amodei N, Lamb RJ. The role of nicotine replacement therapy in early quitting success. Nicotine Tob Res. 2010;12(1):1-10.

19. Romanowich P, Mintz J, Lamb RJ. The relationship between selfefficacy and reductions in smoking in a contingency management procedure. Exp Clin Psychopharmacol. 2009;17(3):139-145.

20. Heil SH, Tidey JW, Holmes HW, et al. A contingent payment model of smoking cessation: Effects on abstinence and withdrawal. Nicotine Tob Res. 2003;5(2):205-213.

21. Mitchell SH. Measures of impulsivity in cigarette smokers and nonsmokers. Psychopharmacology (Berl). 1999;146(4):455-464.

22. Ohmura Y, Takahashi T, Kitamura N. Discounting delayed and probabilistic monetary gains and losses by smokers of cigarettes. Psychopharmacology (Berl). 2005;182(4):508-515.

23. Lamb RJ, Kirby KC, Morral AR, et al. Improving contingency management programs for addiction. Addict Behav. 2004;29(3):507-523.

24. Mueller ET, Landes RD, Kowal BP, et al. Delay of smoking gratification as a laboratory model of relapse: Effects of incentives for not smoking, and relationship with measures of executive function. Behav Pharmacol. 2009;20(5-6):461-473.

25. MacKillop J, Kahler CW. Delayed reward discounting predicts treatment response for heavy drinkers receiving smoking cessation treatment. Drug Alcohol Depend. 2009;104(3):197-203.

26. Krishnan-Sarin S, Reynolds B, Duhig AM, et al. Behavioral impulsivity predicts treatment outcome in a smoking cessation program for adolescent smokers. Drug Alcohol Depend. 2007;88(1):79-82.

27. Jaroni JL, Wright SM, Lerman C, et al. Relationship between education and delay discounting in smokers. Addict Behav. 2004;29(6):1171-1175.

28. Heil SH, Alessi SM, Lussier JP, et al. An experimental test of the influence of prior cigarette smoking abstinence on future abstinence. Nicotine Tob Res. 2004;6(3):471-479.
29. Roll JM, Chudzynski JE. Potential sources of reinforcement and punishment in a drug-free treatment clinic: Client and staff perceptions. $A m J$ Drug Alcohol Abuse. 2005;31(1):21-33.

30. Hanson K, Allen S, Jensen S, et al. Treatment of adolescent smokers with the nicotine patch. Nicotine Tob Res. 2003;5(4):515-526.

31. Munarini E, de Marco C, Rossetti E, et al. Efficacy of pins and diplomas as a reward for long-term smoking cessation. Tumori. 2009;95(3):286-290.

32. Tiede LP, Hennrikus DJ, Cohen BB, et al. Feasibility of promoting smoking cessation in small worksites: An exploratory study. Nicotine Tob Res. 2007;9(S1):S83-S90.

33. Balch GI. Exploring perceptions of smoking cessation among high school smokers: Input and feedback from focus groups. Prev Med. 1998;27(5 Pt 3):A55-A63.

34. Alessi SM, Badger GJ, Higgins ST. An experimental examination of the initial weeks of abstinence in cigarette smokers. Exp Clinical Psychopharmacol. 2004;12(4):276-287.

35. Hughes JR, Hatsukami D. Signs and symptoms of tobacco withdrawal. Arch Gen Psychiatry. 1986;43(3):289-294.

36. Hughes JR, Hatsukami D. Errors in using tobacco withdrawal scale Tob Control. 1998;7(1):92-93.

37. Tiffany ST, Drobes DJ. The development and initial validation of a questionnaire on smoking urges. Br J Addict. 1991;86(11):1467-1476.

38. Tevyaw TO, Colby SM, Tidey JW, et al. Contingency management and motivational enhancement: a randomized clinical trial for college student smokers. Nicotine Tob Res. 2009;11(6):739-749.

39. Lamb RJ, Morral AR, Galbicka G, et al. Shaping reduced smoking in smokers without cessation plans. Exp Clin Psychopharmacol. 2005;13(2):83-92.

40. Lamb RJ, Morral AR, Kirby KC, et al. Contingencies for change in complacent smokers. Exp Clin Psychopharmacol. 2007;15(3):245-255.

41. Lamb RJ, Kirby KC, Morral AR, et al. Shaping smoking cessation in hard-to-treat smokers. J Consult Clin Psychol. 2010;78(1):62-71.

42. Krishnan-Sarin S, Duhig AM, McKee SA, et al. Contingency management for smoking cessation in adolescent smokers. Exp Clin Psychopharmacol. 2006;14(3):306-310.

43. Roll JM, Howard JT. The relative contribution of economic valence to contingency management efficacy: a pilot study. J Appl Behav Anal. 2008;41(4):629-633.

44. Cahill K, Perera R. Competitions and incentives for smoking cessation. Cochrane Database Syst Rev. 2008;(3):CD004307.

45. Donatelle R, Hudson D, Dobie S, et al. Incentives in smoking cessation: status of the field and implications for research and practice with pregnant smokers. Nicotine Tob Res. 2004;6(Suppl 2):S163-S179.

46. Leeks KD, Hopkins DP, Soler RE, Aten A. Chattopadhyay SK, Task Force on Community Preventive Services. Worksite-based incentives and competitions to reduce tobacco use: A systematic review. Am J Prev Med. 2010;38 Supp1 2:S263-S274.

47. Volpp KG, Troxel AB, Pauly MV, et al. A randomized, controlled trial of financial incentives for smoking cessation. $N$ Engl J Med. 2009:360(7):699-709.

48. Hennrikus DJ, Jeffery RW, Lando HA, et al. The SUCCESS project: the effect of program format and incentives on participation and cessation in worksite smoking cessation programs. Am J Public Health. 2002;92(2):274-279.

49. Volpp KG, Gurmankin Levy A, Asch DA, et al. A randomized controlled trial of financial incentives for smoking cessation. Cancer Epidemiol Biomarkers Prev. 2006;15(1):12-18.

50. Reynolds B, Dallery J, Shroff P, et al. A web-based contingency management program with adolescent smokers. J Appl Behav Anal. 2008;41(4):597-601.

51. Alessi SM, Petry NM, Urso J. Contingency management promotes smoking reductions in residential substance abuse patients. $J \mathrm{Appl}$ Behav Anal. 2008;41(4):617-622.

52. Robles E, Crone CC, Whiteside-Mansell L, et al. Voucher-based incentives for cigarette smoking reduction in a women's residential treatment program. Nicotine Tob Res. 2005;7(1):111-117. 
53. Lawrence D, Mitrou F, Zubrick SR. Smoking and mental illness: Results from population surveys in Australia and the United States. BMC Public Health. 2009;(9):285.

54. Lasser K, Boyd JW, Woolhandler S, et al. Smoking and mental illness: A population-based prevalence study. JAMA. 2000;284(20):2606-2610.

55. Tidey JW, O’Neill SC, Higgins ST. Contingent monetary reinforcement of smoking reductions, with and without transdermal nicotine, in outpatients with schizophrenia. Exp Clin Psychoparmacol. 2002;10(3):241-247.

56. Axon RN, Bradford WD, Egan BM. The role of individual time preferences in health behaviors among hypertensive adults: a pilot study. J Am Soc Hypertens. 2009;3(1):35-41.
57. Zhang L, Rashad I. Obesity and time preference: The health consequences of discounting the future. J Biosoc Sci. 2008;40(1):97-113.

58. Gurmankin Levy A, Micco E, Putt M, et al. Value for the future and breast cancer-preventive health behavior. Cancer Epidemiol Biomarkers Prev. 2006;15(5):955-960.

59. Sloan FA, Wang Y. Economic theory and evidence on smoking behavior of adults. Addiction. 2008;103(11):1777-1785.

60. Chapman GB. Short-term cost for long-term benefit: Time preference and cancer control. Health Psychol. 2005;24 Suppl 4:S41-S48.

61. Ferguson BS. Economic modeling of the rational consumption of addictive substances. Subst Use Misuse. 2006;41(4):573-603.

\section{Publish your work in this journal}

Patient Preference and Adherence is an international, peer-reviewed, open access journal focusing on the growing importance of patient preference and adherence throughout the therapeutic continuum. Patient satisfaction, acceptability, quality of life, compliance, persistence and their role in developing new therapeutic modalities and compounds to optimize clinical outcomes for existing disease states are major areas of interest. This journal has been accepted for indexing on PubMed Central. The manuscript management system is completely online and includes a very quick and fair peer-review system. Visit http://www.dovepress.com/ testimonials.php to read real quotes from published authors. 